\title{
Extended thoracic lymph node dissection in robotic-assisted minimal invasive esophagectomy (RAMIE) for patients with superior mediastinal lymph node metastasis
}

\author{
Sylvia van der Horst, Michiel F. G. de Maat, Pieter C. van der Sluis, Jelle P. Ruurda, Richard van Hillegersberg \\ Department of Surgery, University Medical Center Utrecht, Utrecht, The Netherlands \\ Correspondence to: Sylvia van der Horst. Department of Surgery, G04-228, University Medical Center Utrecht, Heidelberglaan 100, 3584 CX, \\ Utrecht, The Netherlands. Email: s.horst-2@umcutrecht.nl.
}

\begin{abstract}
Background: Robot-assisted surgery may have a role in improving oncological outcomes in esophagectomy. Especially in the anatomical areas in the chest that are more difficult to reach in open surgery (including the superior mediastinum). The dexterity of the robotic instruments aid in performing a more extensive nodal dissection and the precision and detailed vision of the robotic system potentially improves staging, oncological outcomes and reduces complications (i.e., recurrent nerve palsy). In this article, we describe our experience and clinical outcomes in patients treated by robot assisted minimal invasive esophagectomy (RAMIE) in cN+ esophageal cancer patients with positive nodes localized in the superior mediastinum.
\end{abstract}

Methods: From May 2007-2018, all patients who had involved nodes by either fluor-18-deoxyglucose positron-emission-tomography-computed tomography (FDG-PET-CT) or endoscopic ultrasound (EUS) + fine needle aspiration (FNA) localized in the superior mediastinum (above level Th4/sternal angle) were identified. Patient characteristics, perioperative data, postoperative clinical outcomes/complications and overall survival were prospectively recorded and retrospectively evaluated.

Results: Forty patients (48\% adenocarcinoma) met our inclusion criteria. All patients underwent a three-stage procedure with cervical anastomosis and $90 \%$ of the patients underwent neoadjuvant chemoradiotherapy. Mortality occurred in three patients $(7.5 \%)$, of which two were caused by severe acute respiratory distress syndrome (ARDS). The most frequent complications were pneumonia (25\%), chylothorax (20\%), anastomotic leakage (17.5\%) and vocal cord paralysis (17.5\%) which was grade 1 in $72 \%$ of the patients. Radicality rate (R0 resection) was $98 \%$ and the average lymph node yield was 24 (range, 9-57). Median overall and disease-free survival was 26 and 17 months, respectively.

Conclusions: RAMIE for esophageal cancer patients with node positive disease in the superior mediastinum is associated with increased mortality/morbidity. Oncological outcome showed excellent lymph node yield, R0 rate and survival was equal compared to patients with lower mediastinal node positive disease.

Keywords: Lymph node dissection; robotic; minimally invasive esophagectomy (MIE); mediastinal lymph nodes; metastasis

Submitted Oct 02, 2018. Accepted for publication: Dec 11, 2018.

doi: $10.21037 /$ acs.2019.01.04

View this article at: http://dx.doi.org/10.21037/acs.2019.01.04

\section{Introduction}

Esophageal cancer is the sixth ranking cause of cancerrelated mortality world-wide and due to its complex and highly invasive treatment or cancer of the gastro-esophageal junction (GEJ), it is associated with a major impact on quality of life for patients (1-3). Early symptoms are subtle and therefore the majority of the patients are diagnosed at an advanced stage $(2,3)$. The cTNM stage in the majority of patients ( $>70 \%)$ with curative disease is cT3-4N1-2 $(2,3)$. Complete lymphadenectomy during surgical resection is 
important for adequate nodal staging. The number of nodes removed has been shown to improve survival in esophageal cancer patients (4). The extent of lymphadenectomy during oncological esophagectomy however, is not standardized (4). The paratracheal stations [2,4,5] (5) are often avoided as this area is difficult to reach and dissection is considered technically challenging (6-9). Therefore, upper mediastinal dissection is associated with an increased risk of recurrent laryngeal nerve (RLN) palsy, vagal nerve damage and damage of the membranous part of the trachea (6-9). However, in a significant number of patients, preoperative imaging [fluor-18-deoxyglucose positron-emissiontomography-computed tomography (FDG-PET-CT) or endoscopic ultrasonography] shows lymph node metastasis in the superior mediastinum. Precise lymphadenectomy above the level of the of the azygous vein arcus in these patients is important to offer optimal treatment (6-9).

Open surgery is the gold standard in oncological esophagectomy, including incisional access to $2-3$ compartments, of which the thoracic phase has the highest impact on postoperative morbidity $(10,11)$. Thoracoscopic robot-assisted minimally invasive surgery has the advantage of reducing invasiveness combined with improved visual and dissection capabilities over open surgery $(12,13)$. Results of the recently published ROBOT trial showed improved clinical outcomes with reduced surgical and cardiopulmonary complication rates, reduced pain and improved functional outcomes of the robot-assisted procedure over open surgery $(14,15)$. Furthermore, in open surgery, access to the thoracic cavity is hampered by the ribs and scapula, especially where a detailed view of the thoracic aperture is needed in patients who need lymphadenectomy in the superior mediastinum $(12,13)$. For this patient group in particular, robot-assisted minimally invasive esophagectomy (RAMIE) could be very useful in achieving a complete nodal harvest with a minimal risk of complications $(12,13)$.

The objective of this study was to describe the clinical outcomes of esophageal cancer patients with lymph node metastasis in the superior mediastinum operated in our hospital with RAMIE. We focus on complication rates (in particular recurrent nerve damage), histopathological results and oncological outcomes.

\section{Methods}

\section{Patients}

From May 2007-Sept 2018, patients without evidence of distant metastases or cT4b disease that underwent curative robot-assisted thoraco-laparoscopic esophageal resection were identified in our prospectively maintained database. Patients were selected for this study when preoperative staging showed evidence of nodal disease in the superior mediastinum by PET-CT or endoscopic ultrasound with positive cytology on fine-needle aspiration. All patients were discussed in a multidisciplinary tumor board before treatment and underwent routine staging including gastroduodenoscopy, endoscopic ultrasonography, ultrasonography of the neck and a (PET) CT-scan of the thorax-abdomen. When needed, endobronchial ultrasonography was performed with FNA of possible involved lymph nodes. Institutional review board approval and a waiver for informed consent requirement was obtained for this study (MERC UMC Utrecht ID041, approval number 13-061/C).

\section{Preoperative data}

Baseline characteristics included sex, age, BMI, medical history and clinical tumor type. cTNM (16) staging was based on the above mentioned staging modalities and was agreed on during the multidisciplinary tumor board meetings. Neoadjuvant treatment was allocated to patients according to the Dutch oncological guidelines [online at https://www.oncoline.nl/oesofaguscarcinoom (in Dutch)]. Before 2012, patients with adenocarcinoma were treated with peri-operative triplet systemic treatment (epirubicin, cisplatin and infused 5-fluorouracil) (17). Patients with squamous cell cancer were treated with the CROSS schedule (carboplatin and paclitaxel weekly with 41.4 Gy radiation dose) and this was also administered to patients with adenocarcinoma after May 2012 (18).

\section{Surgical procedure}

All patients were operated by thoracolaparoscopic RAMIE (da Vinci robotic system Intuitive Surgical Inc., Sunnyvale, California, USA) with two field lymphadenectomies. The surgical procedure was described in detail previously $(12,13)$. For the thoracic approach, the patient was positioned in the left lateral decubitus position, slightly tilted towards prone position. Mediastinal lymphadenectomy was standardized and included the bilateral paratracheal (station 2), tracheobronchial (station 4), aorto-pulmonary window (station 5), carinal (station 7), periesophageal (station 8) and pulmonary ligament (station 9) lymph nodes. 


\begin{tabular}{lll}
\hline \multicolumn{2}{l}{ Table 1 Uniform pneumonia score } & \\
\hline Parameter & Range & Score \\
\hline Temperature $\left({ }^{\circ} \mathrm{C}\right)$ & $\geq 36.1$ and $\leq 38.4$ & 0 \\
& $\geq 38.5$ and $\leq 38.9$ & 1 \\
& $\geq 39$ and $\leq 36.0$ & 2 \\
\hline $\begin{array}{l}\text { Leucocyte count, } \\
\times 10^{9} / \mathrm{l}\end{array}$ & $>4.0$ and $\leq 11.0$ & 0 \\
\hline $\begin{array}{l}\text { Pulmonary radiography } \\
\text { No infiltrate }\end{array}$ & 1 \\
& Diffused (or patchy) infiltrate & 1 \\
& Well-circumscribed infiltrate & 2 \\
\hline $\begin{array}{l}\text { A sum score of } 2 \text { point or higher, of which at least 1 point assigned } \\
\text { due to infiltrative findings on radiography, indicates treatment of } \\
\text { pneumonia (20). }\end{array}$
\end{tabular}

Table 2 Patient demographics and tumor characteristics $(\mathrm{n}=40)$

\begin{tabular}{|c|c|c|c|}
\hline Variable & n (\%) & Median & Range \\
\hline Age (years) & & 67 & $51-80$ \\
\hline \multicolumn{4}{|l|}{ Gender } \\
\hline Male & $15(37.5)$ & & \\
\hline Female & $25(62.5)$ & & \\
\hline BMI $\left(\mathrm{kg} / \mathrm{m}^{2}\right)$ & & 25 & $18-41$ \\
\hline \multicolumn{4}{|l|}{ Co-morbidity } \\
\hline Vascular & $16(40.0)$ & & \\
\hline Cardiac & $12(30.0)$ & & \\
\hline Pulmonary & $10(25.0)$ & & \\
\hline Diabetes & $5(12.5)$ & & \\
\hline Oncologic & $6(15.0)$ & & \\
\hline \multicolumn{4}{|l|}{ Intoxications } \\
\hline Smoking & $10(25.0)$ & & \\
\hline Alcohol & $26(65.0)$ & & \\
\hline \multicolumn{4}{|l|}{ ASA score } \\
\hline 1 & $6(15.0)$ & & \\
\hline 2 & $25(62.5)$ & & \\
\hline 3 & 9 (22.5) & & \\
\hline $\begin{array}{l}\text { ASA, Americ } \\
\text { body mass in }\end{array}$ & ty of Ane & iologists & ication; \\
\hline
\end{tabular}

The abdominal phase was performed by conventional laparoscopy and standard lymphadenectomy included stations 9 (celiac trunk), 8a (anterior of the hepatic artery) and $11 \mathrm{p}$ (proximal splenic artery) (5). The gastric conduit was performed with a stapler device, brought up to the neck and an end-to-side anastomosis was made with a single layer, hand-sewn running suture. Details of the surgical procedure (i.e., operation time, blood loss and intraoperative complications) were registered prospectively.

\section{Postoperative outcome data}

Postoperative tube feeding by an intraoperatively placed jejunostomy was done in case of severe or unrecovered weight loss. Patients were extubated on the day of surgery and monitored in the intensive care unit (ICU) ward the first night. Registration of the postoperative outcomes and complications was done prospectively at a weekly consensus meeting with the attending surgeons, physician assistants and researchers. At this meeting, complication severity was agreed upon using the Modified Clavien Dindo Classification (19). Pneumonia was scored using the Unified Pneumonia Score (Table 1) (20,21). Mortality was registered as in-hospital, 30- and 90-day. Follow-up was standardized according to the Dutch guidelines up to 5 years postoperatively.

\section{Statistical analysis}

Statistical analysis was performed using SPSS version 22. Mostly descriptive data are reported in this study of the patient group and no comparative analyses between groups were performed. Distribution of nodal yield is presented in a box-plot. Survival duration was calculated as duration from the day of surgery to death or last day of follow-up. Disease-free interval was calculated from the day of surgery to the day of definitive diagnosis of recurrent disease.

\section{Results}

From May 2007 to August 2018, 604 patients underwent elective esophagectomy for esophageal cancer in our hospital. A total of 40 consecutive patients were identified with lymph node metastases in the superior mediastinum. Baseline characteristics are given in Tables 2,3. The majority of patients were male and most patients had 


\begin{tabular}{|c|c|}
\hline Variable & n (\%) \\
\hline \multicolumn{2}{|l|}{ Clinical T stage } \\
\hline cT1 & $2(5.0)$ \\
\hline сT2 & $5(12.5)$ \\
\hline сT3 & $30(75.0)$ \\
\hline cT4a & $5(12.5)$ \\
\hline \multicolumn{2}{|l|}{ Clinical N stage } \\
\hline cNO & 0 \\
\hline $\mathrm{cN} 1$ & $17(42.5)$ \\
\hline cN2 & $19(47.5)$ \\
\hline cN3 & $4(10.0)$ \\
\hline \multicolumn{2}{|l|}{ Tumour type } \\
\hline Adenocarcinoma & $19(47.5)$ \\
\hline Squamous cell carcinoma & $21(52.5)$ \\
\hline \multicolumn{2}{|l|}{ Site of tumour } \\
\hline Upper esophageal (18-24 cm) & $12(30.0)$ \\
\hline Mid esophageal (24-32 cm) & $14(35.0)$ \\
\hline Lower esophageal/GEJ (>32 cm) & $14(35.0)$ \\
\hline \multicolumn{2}{|l|}{ Neoadjuvant treatment } \\
\hline No therapy & $4(10.0)$ \\
\hline Chemotherapy & $3(7.5)$ \\
\hline Chemoradiotherapy & $29(72.5)$ \\
\hline Extended chemoradiotherapy & $4(10.0)$ \\
\hline
\end{tabular}

cT3N1-2 tumors that were localized in the middle third of the esophagus. Neoadjuvant therapy was given in $90 \%$ of the patients and consisted of chemoradiotherapy in $83 \%$ of the patients and chemotherapy in all others. In 4 patients, no neo-adjuvant treatment was administered due to renal insufficiency, cardiac disease, patient refusal and in one patient, the reason was unknown. Patients receiving chemoradiotherapy were mostly treated according to the CROSS schedule (29 patients), but extended chemoradiotherapy was administered to 4 patients (10\%) for various reasons. One patient was already treated in another hospital and was initially deemed irresectable due to the proximal location of the primary tumor. After showing a good response, the patient came to our center

\begin{tabular}{|c|c|c|c|}
\hline Variable & $\mathrm{n}(\%)$ & Median & Range \\
\hline Uncomplicated procedures & $10(25.0)$ & & \\
\hline Pneumonia (UPS) & $11(27.5)$ & & \\
\hline Anastomotic leakage & $7(17.5)$ & & \\
\hline Intrathoracic manifestations & $7(17.5)$ & & \\
\hline Necrosis gastric tube & $1(2.5)$ & & \\
\hline Chylothorax & $10(25.0)$ & & \\
\hline Vocal cord paralysis & 7 (17.5) & & \\
\hline Temporary & $5(12.5)$ & & \\
\hline \multicolumn{4}{|l|}{ Cardiac } \\
\hline Atrial fibrillation & $6(15.0)$ & & \\
\hline Thrombo embolic event & $1(2.5)$ & & \\
\hline In-hospital death & $3(7.5)$ & & \\
\hline ICU stay (days) & & 1 & $1-95$ \\
\hline Hospital stay (days) & & 13 & $7-124$ \\
\hline
\end{tabular}

and repeated imaging showed that a negative margin could be obtained and the patient went to surgery. Two patients were not considered eligible for surgery elsewhere because of the nodal involvement in the superior mediastinum. After completion of a long-course schedule and showing a good radiological response, they we discussed in our multidisciplinary tumor board and surgical resection was offered. The final patient underwent extended chemoradiotherapy because of radiographical signs of contact of an involved node with the membranous part of the trachea.

\section{Postoperative outcome data}

Details of the clinical postoperative outcomes are listed in Table 4. In 10 (25\%) of the patients an uncomplicated outcome was achieved. Of the remaining 30 patients, 17 (43\%) had Clavien Dindo grade I or II complications. Pneumonia was encountered most frequently and occurred in 11 patients (28\%). RLN palsy rates occurred in seven patients $(18 \%)$ and this recovered without intervention (Grade 1) in five patients. Anastomotic leakage occurred in seven patients. All were surgically drained. In total, three patients died in the hospital postoperatively. One 


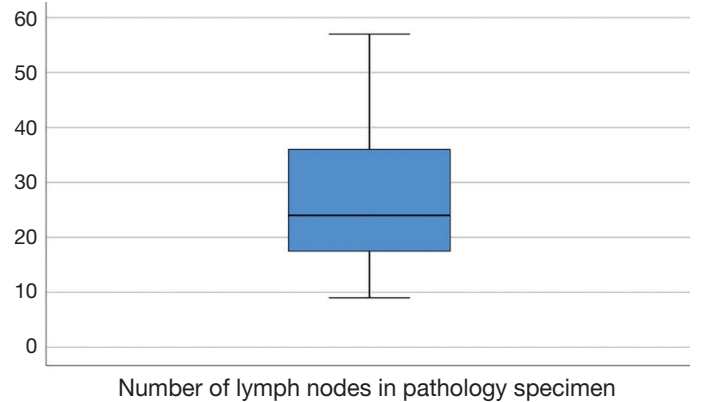

Figure 1 Boxplot of the distribution of lymph nodes retrieved from the resection specimen.

\begin{tabular}{lccc}
\multicolumn{2}{l}{ Table 5 Histopathological data $(\mathrm{n}=40)$} & & \\
\hline Variable & $\mathrm{n}(\%)$ & Median & Range \\
\hline Radicality & & \\
R0 & $39(97.5)$ & & \\
R1 & $1(2.5)$ & & \\
Retrieved lymph node & & 24 & $9-57$ \\
Positive lymph node & & 0 & $0-18$ \\
\hline
\end{tabular}

patient developed acute respiratory distress syndrome in combination with anastomotic leakage which resulted into an extensive period in the ICU for respiratory support. After prolonged weaning he was transferred to the ward and in the waiting period for admittance to a rehabilitation clinic, he developed acute respiratory failure. Due to treatment restrictions, he was not readmitted to the ICU and died. A second patient with COPD GOLD III also developed ARDS without signs of anastomotic leakage. Extracorporeal membrane oxygenation (ECMO) was started, however, the patient died because of insufficient pulmonary reserves. A third patient developed severe sepsis with multi-organ failure due to leakage and abscesses of the jejunostomy. Neither of these patients received extended chemoradiotherapy before surgery.

\section{Oncological outcomes}

All but one patient (cT4a) had a radical resection (R0) and the radicality rate therefore was $98 \%$. The patient with a $\mathrm{R} 1$ resection had a tumor localized from $26-36 \mathrm{~cm}$ and at the operation, the arcus of the azygous vein appeared to be involved (T4) and was filled with thrombus. The arcus was resected en-bloc with the tumor. The pathology report showed a tumor regression grade Mandard 4 (residual cancer outgrowing fibrosis) (22) and the positive margin was at the venous transection. The thrombus that was sent separately for pathology investigation was positive for adenocarcinoma. The number of resected nodes on average was 26 (95\% CI, 23-30), see also Figure 1, Table 5. At the time of this analysis, 24 patients $(60 \%)$ were deceased and $48 \%$ had recurrent disease. Median survival was 26 months at median duration of follow-up of 7 months.

\section{Discussion}

This study describes consecutive series of a specific group of esophageal cancer patients with clinical metastasis $(\mathrm{cN}+)$ to the superior mediastinal nodes. Results showed that RAMIE with nodal dissection in the superior mediastinum had excellent lymph node yields and R0 rate, with significant but acceptable complication rates. The in-hospital mortality was increased compared to mortality rates reported in the ROBOT trial (4\%) (15). Two patients who died developed ARDS, which in one case was accompanied by anastomotic leakage. In the literature, ARDS after esophagectomy is reported in $13 \%$ of the cases and is associated with increased mortality $(20 \%)(23,24)$. Preoperative respiratory conditions, such as COPD GOLD III (as in one of the deceased patients in our series), as well as intraoperative factors (i.e., administered oxygen percentage, use of vasopressors) are associated with the occurrence of ARDS $(23,24)$. As a preventive measurement, we administer methylprednisolone prior to the start of the thoracic phase of the operations in all patients $(1 \mathrm{mg} / \mathrm{kg}$ body weight as is suggested by many groups $(25-27)$. Whether this specific group of patients with radiotherapy of the superior mediastinum is prone to develop ARDS is unclear. One patient died because of complications of the jejunostomy and this cannot be directly related to the more complex thoracic mediastinal surgery. In a systematic review focusing on esophageal cancer surgery, minor jejunostomy related complications are seen frequently $(13-38 \%)$ however mortality is rare $(0.0-0.5 \%)(28)$.

It is important to mention that our center is a tertiary referral center for complex cases, as is illustrated by the patients who are included in our series that underwent extended chemo-radiotherapy. Involvement of high mediastinal nodes is often used as an argument to refrain from surgical resection in the referring hospital. The results of this study show that a curative, minimal invasive 
treatment is feasible for these patients by performing RAMIE.

An important endpoint for this study's analysis was recurrent nerve palsy rate as this is a complication that is specifically associated with paratracheal nodal dissection in the superior mediastinum. A previous study on 40 patients show palsy rates in $20 \%$ of patients, however, they describe skeletonization of the RLN in a subgroup and this increased palsy rates to $32 \%$ compared to $6 \%$ in the group where only tissue dorsal of the RLN was taken (6). A subsequent study describes a significant learning curve effect for RLN palsy rates and the analyses of the study showed that rates drop from $55 \%$ to $0 \%$ after performing 20 cases (7). In the current study the RLN palsy rate was $18 \%$ and most cases were temporary and required conservative management only (Grade 1) (29). The rate of injuries in patients with $\mathrm{cN}+$ disease in the superior mediastinum was markedly increased compared to the palsy rate in patients that were included in the ROBOT trial which was $9 \%(15)$. In this trial, the primary tumor was located at the distal third of the esophagus or GE junction in $89 \%$ of patients and patients with high positive nodes were excluded. The doubled RLN palsy rate in the current patient series can be explained by the complexity of the cases and the challenging and more extensive dissection in the superior mediastinum. The effect of the chemoradiotherapy on the tissues surrounding the positive nodes often results in a fibrotic reaction which compromises the natural planes and impedes separation of the nodes from vital structures.

The importance of extensive nodal dissection in esophageal cancer surgery is supported by comprehensive evidence in the literature. A population-based Cohort Study in the Netherlands including 2,698 patients showed that a higher lymph node yield was significantly associated with improved overall survival, indicating the therapeutic value of extended lymphadenectomy during esophagectomy (4). Furthermore, a high lymph node yield was associated with favorable hazard ratios across all subgroups, including both squamous cell carcinoma and adenocarcinoma patients, cN0 and $\mathrm{cN}+$ patients, transthoracic and trans-hiatal approaches, and both ypN0 and ypN+ patients. In this study the overall average nodal yield was 16 for all groups and 19 for patients operated by a transthoracic approach (4). The average number of nodes dissected in our study was therefore markedly higher compared to the data collected by the Netherlands national Cancer Registry.

The occurrence of superior mediastinal lymph nodes metastases ranges from $4-28 \%$ in mixed patient cohorts containing adeno-as well as squamous cell carcinoma in Western world study populations $(13,30,31)$. For patients with mid-thoracic squamous cell carcinomas in Asia, the lymph node metastasis rates of the thoracic left para-RLN (1, 2, and $4 \mathrm{~L}$ zones) and right para-RLN (1R zone) were $30 \%$ and $28 \%$ in 128 cases, respectively. The metastasis rates of the $2 \mathrm{R}, 4 \mathrm{R}$, and 5 zones were $5 \%, 4 \%$, and $5 \%$, respectively (9). These results show that in squamous cell cancers an upper mediastinal lymph node dissection including the thoracic left para-RLN, the right para-RLN and paratracheal lymph nodes is pivotal to obtain a radical resection and reach favorable overall survival. A study that specifically looked into the most prevalent tumor type in the Western world, namely adenocarcinoma of the distal third of the esophagus or the GE junction demonstrated that about $10 \%$ of the patients have positive nodes in the proximal chest (including the carinal and hilar stations 7 and 10) (32). Therefore, we advise standard lymphadenectomy of the superior mediastinum be performed in all cases.

This is a relatively small case series from our center, which has extensive experience in performing RAMIE with complete 2-field lymph node dissection. In the Western population however, no larger series exist for this specific patient group to our knowledge, and therefore we think the results are of interest for clinicians considering a robotic approach for esophagectomy. Adopting RAMIE requires step-wise training and learning curve effects must be taken into account (33). Therefore we advise a carefully designed proctoring program and to start with regular cases without proximal $\mathrm{cN}+$ nodes Mediastinal nodal dissection can also be performed with conventional video-assisted thoracoscopic surgery (VATS) and reported results show favorable oncological and clinical outcomes $(9,34,35)$. Our center does not have experience with VATS lymphadenectomy in the superior mediastinum as early-on we adopted robotics during the switch to minimal invasive procedures. In general VATS lymphadenectomy in the thoracic aperture is considered technically challenging where the robot provides a stable, enlarged three-dimensional view and the articulating instruments with tremor filter, facilitating meticulous dissection (36). Therefore RAMIE is of added value for complex cases such as patients with nodal disease in the superior mediastinum. The results of this study support this.

\section{Acknowledgements}

None. 


\section{Footnote}

Conflicts of Interest: JP Ruurda and R van Hillegersberg are proctors for Intuitive Surgical Inc.

Ethical Statement: Institutional review board approval and a waiver for informed consent requirement was obtained for this study (MERC UMC Utrecht ID041, approval number $13-061 / \mathrm{C})$.

\section{References}

1. Ferlay J, Soerjomataram I, Dikshit R, et al. Cancer incidence and mortality worldwide: sources, methods and major patterns in GLOBOCAN 2012. Int J Cancer 2015;136:E359-86.

2. Enzinger PC, Mayer RJ. Esophageal cancer. N Engl J Med 2003;349:2241-52.

3. Pennathur A, Gibson MK, Jobe BA, et al. Oesophageal carcinoma. Lancet 2013;381:400-12.

4. Visser E, van Rossum PS, Ruurda JP, et al. Impact of lymph node yield on overall survival in patients treated with neoadjuvant chemoradiotherapy followed by esophagectomy for cancer - a population-based cohort study in the Netherlands. Ann Surg 2017;266:863-9.

5. Naruke T, Tsuchiya R, Kondo H, et al. Lymph node sampling in lung cancer: how should it be done? Eur J Cardiothorac Surg 1999;16:S17-24.

6. Kim DJ, Park SY, Lee S, et al. Feasibility of a robotassisted thoracoscopic lymphadenectomy along the recurrent laryngeal nerves in radical esophagectomy for esophageal squamous carcinoma. Surg Endosc 2014;28:1866-73.

7. Park SY, Kim DJ, Kang DR, et al. Learning curve for robotic esophagectomy and dissection of bilateral recurrent laryngeal nerve nodes for esophageal cancer. Dis Esophagus 2017;30:1-9.

8. Chao YK, Hsieh MJ, Liu YH, et al. Lymph Node Evaluation in Robot-Assisted Versus Video-Assisted Thoracoscopic Esophagectomy for Esophageal Squamous Cell Carcinoma: A Propensity-Matched Analysis. World J Surg 2018;42:590-8.

9. Lv WQ, Wei WZ, Wu WB, et al. Significance of Upper Mediastinal Lymph Node Dissection with Video-Assisted Thoracic Surgery in the Treatment of Middle Thoracic Esophageal Carcinoma. J Laparoendosc Adv Surg Tech A 2017;27:1061-4.

10. Hulscher JB, van Sandick JW, de Boer AG, et al. Extended transthoracic resection compared with limited transhiatal resection for adenocarcinoma of the esophagus. $\mathrm{N}$ Engl J Med 2002;347:1662-9.

11. Haverkamp L, Seesing MF, Ruurda JP, et al. Worldwide trends in surgical techniques in the treatment of esophageal and gastroesophageal junction cancer. Dis Esophagus 2017;30:1-7.

12. van Hillegersberg R, Boone J, Draaisma WA, et al. First experience with robot-assisted thoracoscopic esophagolymphadenectomy for esophageal cancer. Surg Endosc 2006;20:1435-9.

13. Boone J, Schipper ME, Moojen WA, et al. Robot-assisted thoracoscopic oesophagectomy for cancer. Br J Surg 2009;96:878-86.

14. van der Sluis PC, Ruurda JP, van der Horst S, et al. Robot-assisted minimally invasive thoraco-laparoscopic esophagectomy versus open transthoracic esophagectomy for resectable esophageal cancer, a randomized controlled trial (ROBOT trial). Trials 2012;13:230.

15. van der Sluis PC, van der Horst S, May AM, et al. Robot-assisted Minimally Invasive Thoracolaparoscopic Esophagectomy Versus Open Transthoracic Esophagectomy for Resectable Esophageal Cancer: A Randomized Controlled Trial. Ann Surg 2019;269:621-30.

16. Sobin LH, Gospodarowicz MK, Wittekind C. TNM Classification of Malignant Tumors, 7th edition. Oxford: Wiley-Blackwell, 2009.

17. Cunningham D, Allum WH, Stenning SP, et al. Perioperative chemotherapy versus surgery alone for resectable gastroesophageal cancer. $\mathrm{N}$ Engl J Med 2006;355:11-20.

18. van Hagen P, Hulshof MC, van Lanschot JJ, et al. Preoperative chemoradiotherapy for esophageal or junctional cancer. N Engl J Med 2012;366:2074-84.

19. Dindo D, Demartines N, Clavien P. Classification of Surgical Complications A New Proposal With Evaluation in a Cohort of 6336 Patients and Results of a Survey. Ann Surg 2004;240:205-13.

20. van der Sluis PC, Verhage RJ, van der Horst S, et al. A new clinical scoring system to define pneumonia following esophagectomy for cancer. Dig Surg 2014;31:108-16.

21. Weijs TJ, Seesing MF, van Rossum PS, et al. Internal and External Validation of a multivariable Model to Define Hospital-Acquired Pneumonia After Esophagectomy. J Gastrointest Surg 2016;20:680-7.

22. Mandard AM, Dalibard F, Mandard JC, et al. Pathologic assessment of tumor regression after preoperative chemoradiotherapy of esophageal carcinoma. 
Clinicopathologic correlations. Cancer 1994;73:2680-6.

23. Paul DJ, Jamieson GG, Watson DI, et al. Perioperative risk analysis for acute respiratory distress syndrome after elective oesophagectomy. ANZ J Surg 2011;81:700-6.

24. Tandon S, Batchelor A, Bullock R, et al. Peri-operative risk factors for acute lung injury after elective oesophagectomy. Br J Anaesth 2001;86:633-8.

25. Park SY, Lee HS, Jang HJ, et al. Efficacy of intraoperative, single-bolus corticosteroid administration to prevent postoperative acute respiratory failure after oesophageal cancer surgery. Interact Cardiovasc Thorac Surg 2012;15:639-43.

26. Shimada H, Ochiai T, Okazumi S, et al. Clinical benefits of steroid therapy on surgical stress in patients with esophageal cancer. Surgery 2000;128:791-8.

27. Engelman E, Maeyens C. Effect of preoperative singledose corticosteroid administration on postoperative morbidity following esophagectomy. J Gastrointest Surg 2010;14:788-804.

28. Berkelmans GH, van Workum F, Weijs TJ, et al. The feeding route after esophagectomy: a review of literature. J Thorac Dis 2017;9:S785-91.

29. Low DE, Alderson D, Cecconello I, et al. International Consensus on Standardization of Data Collection for Complications Associated With Esophagectomy: Esophagectomy Complications Consensus Group (ECCG). Ann Surg 2015;262:286-94.

30. van der Sluis PC, Ruurda JP, Verhage RJ, et al. Oncologic
Long-Term Results of Robot-Assisted Minimally Invasive Thoraco-Laparoscopic Esophagectomy with Two-Field Lymphadenectomy for Esophageal Cancer. Ann Surg Oncol 2015;22:S1350-6.

31. Parry K, Haverkamp L, Bruijnen RC, et al. Surgical treatment of adenocarcinomas of the gastro-esophageal junction. Ann Surg Oncol 2015;22:597-603.

32. Anderegg MC, Lagarde SM, Jagadesham VP, et al. Prognostic Significance of the Location of Lymph Node Metastases in Patients With Adenocarcinoma of the Distal Esophagus or Gastroesophageal Junction. Ann Surg 2016;264:847-53.

33. van der Sluis PC, Ruurda JP, van der Horst S, et al. Learning Curve for Robot-Assisted Minimally Invasive Thoracoscopic Esophagectomy: Results From 312 Cases. Ann Thorac Surg 2018;106:264-71.

34. Ninomiya I, Osugi H, Fujimura T, et al. Thoracoscopic esophagectomy with extended lymph node dissection in the left lateral position: technical feasibility and oncologic outcomes. Dis Esophagus 2014;27:159-67.

35. Ninomiya I, Okamoto K, Fujimura T, et al. Oncologic outcomes of thoracoscopic esophagectomy with extended lymph node dissection: 10-year experience from a single center.World J Surg 2014;38:120-30.

36. Ruurda JP, van der Sluis PC, van der Horst S, et al. Robotassisted minimally invasive esophagectomy for esophageal cancer: A systematic review. J Surg Oncol 2015;112:257-65.
Cite this article as: van der Horst $\mathrm{S}$, de Maat MF, van der Sluis PC, Ruurda JP, van Hillegersberg R. Extended thoracic lymph node dissection in robotic-assisted minimal invasive esophagectomy (RAMIE) for patients with superior mediastinal lymph node metastasis. Ann Cardiothorac Surg 2019;8(2):218225. doi: 10.21037/acs.2019.01.04 